Reprod. Nutr. Dévelop., 1984, 24 (6), 865-886.

\title{
Modifications ultrastructurales des cellules germinales femelles au cours de la prophase méiotique chez le rat : étude particulière des membranes après cryofracture.
}

\author{
Jacqueline BÉZARD, P. MAULÉON
}

Station de Physiologie de la Reproduction, I.N.R.A., Nouzilly, 37380 Monnaie, France.

Summary. Ultrastructural modifications of female rat germ cells during the meiotic prophase, with special emphasis on the membranes after cryofracture.

Ultrastructural changes in the nuclear and cytoplasmic elements in the germ cells of female rats were followed before meiotic prophase (15.50 days post-coïtum and 17.25 days post-coïtum) and during it (17.75 days post-coitum to birth). We observed :

- modifications in the nuclear envelope which was thick during the oogonial stage, becoming thinner when the chromosomes entered preleptotene stage. The thinning of the envelope was due to the disappearance of the chromatin material lining it ;

- variations in the number and distribution of germ cell nuclear pores according to stage ; the pores were first scattered in small clusters of 6 to 8 over the entire nuclear membrane. From the preleptotene to zygotene stage, these clusters enriched in pores to form large areas. Finally, in the pachytene and diplotene stages, clusters of more than 100 pores were seen :

- nucleolar fragmentation from the preleptotene stage, followed by the formation of a new active nucleole in the diplotene;

- polarization of the mitochondria in the oldest oogonia just before the beginning of meiotic prophase. This polarization disappeared after the onset of the meiotic processes, then appeared again near the developing Golgi apparatus at the end of the pachytene stage ;

- the formation of large gap junctions and numerous bands of tight junctions between the somatic cells; these formations contrasted with small gap junctions, and the tight junctions became scarce just before the meiotic process began.

These observations, as well as those concerning nuclear pore distribution were made using the cryofracture technique.

\section{Introduction.}

Au cours de la méïose des cellules germinales chez le rat, nous avons remarqué sur coupes semi-fines en dehors des modifications nucléaires également identifiables avec cette technique:

- un changement dans l'aspect de l'enveloppe nucléaire, 
- des phénomènes de polarisation de l'appareil de Golgi et des mitochondries, précédant immédiatement l'entrée en méïose et antérieurs au stade de repos du dictyotène,

- la quasi-disparition du nucléole (Bézard et Mauléon, 1984).

En vérifiant la validité de ces événements en microscopie électronique, nous avons apporté des informations qui montrent que le changement d'arrangement des chromosomes est associé à une modification des échanges intra et inter cellulaires. Ce sont des examens de répliques des membranes après cryofracture qui, en localisant les pores nucléaires des cellules germinales ou en mettant en évidence les liaisons très particulières entre cellules somatiques, suggèrent cette évolution des échanges, phénomène non encore décrit à ce jour.

\section{Matériel et méthodes}

Les rats femelles Wistar utilisés sont de même provenance et soumis aux mêmes conditions de prélèvement que les animaux précédemment décrits dans Bézard et Mauléon (1984). Les âges des ovaires au moment du prélèvement ont été choisis afin de réduire au minimum la diversité des types nucléaires présents dans les cellules germinales et d'obtenir le pourcentage le plus élevé du type "dominant " (tabl. 1 : dans Bézard et Mauléon, 1984). De ce fait, les abattages ont été réalisés à $15,25 \mathrm{j}$ post-coïtum, $17,25 \mathrm{j}$ p.c., $17,75 \mathrm{j}$ p.c., 18,25 j p.c., $19,25 \mathrm{j}$ p.c., $21,25 \mathrm{j}$ p.c., $\mathrm{J}_{0}$ (naissance), 1 et $4 \mathrm{j}$ post-natal.

Après une anesthésie à l'éther des rates gestantes et dissection rapide des embryons femelles, les ovaires de ces derniers ont été placés à $4{ }^{\circ} \mathrm{C}$ dans le mélange fixateur de glutaraldéhyde à $2,5 \%$ dans le tampon cacodylate $0,1 \mathrm{M}, \mathrm{pH}$ 7,3 pendant 1 à $3 \mathrm{~h}$, puis rincés à la même température dans ce même tampon.

Après inclusion dans l'Epon, les ovaires droits ont été réservés pour l'examen de coupes semi-fines et ultrafines. Les résultats de l'étude en coupes semi-fines ont été présentés dans l'article de Bézard et Mauléon (1984). Après repérage sur

FIG. 1a et 1b. - Ovogonie $(17,25$ j p.c. $)$. Chromatine dense et homogène, condensation de matériel tout le long de l'enveloppe nucléaire (EN), nucléole volumineux et réticulé (Nu), appareil de Golgi $(G o)$ proche des mitochondries $(\mathrm{m})$, pores nucléaires dispersés sur l'enveloppe (PN). $X: 6000$.

FIG. $2 a$ et $2 b$. - Préleptotène $(17,75$ j p.c.). Amas de chromatine épars, pores nucléaires (PN) répartis en petits groupes sur l'enveloppe nucléaire fine (EN), nucléole fragmentaire (Nu), mitochondries sphériques dispersées $(\mathrm{m})$. $X: 5000$.

FIG. 3a et $3 \mathrm{~b}$. - Leptotène $(18,25$ j p.c.). Filaments chromosomiques fins et courts ( $F C$ ), groupes de pores nucléaires $(P)$, enveloppe nucléaire fine $(E N)$, fragments de nucléoie $(\mathrm{Nu})$, mitochondries (m). $X: 4800$. 
coupes semi-fines des cellules germinales à un stade précis, les observations en microscopie électronique ont été faites sur la coupe suivante et font l'objet de cet article.

Les ovaires gauches ont été traités pour l'obtention de répliques membranajres après cryofracture. Ces ovaires ont été transférés dans un bain cryoprotecteur de glycérol à $10 \%$ dans un tampon cacodylate, puis à $20 \%$, pendant $1 \mathrm{~h}$ à la température du laboratoire, selon la technique mise au point par Escaig et Nicolas (1976). L'appareil de cryofracture utilisé a été le "Cryofact 190 » de ReichertJung.

Un dépôt, par évaporation de platine, a été réalisé à la surface de la cassure pour constituer la réplique ; une évaporation de carbone a consolidé les moulures de platine et donné ainsi des répliques complètes.

Le matériel biologique a été digéré par un bain d'eau de Javel concentrée pendant $5 \mathrm{~h}$. Les répliques ont été rincées très soigneusement à l'eau distillée et récupérées sur des grilles de 600 mesh.

\section{Résultats.}

\section{Description ultrastructurale des stades de la prophase méïotique.}

1. Ovogonie (PI. 1-1a et $1 \mathrm{~b} ; 3-1$ et 2 ). - Les larges noyaux sphériques des " ovogonies » ont une chromatine dense aux électrons et homogène. Du matériel chromatinien plus dense est disposé tout le long de l'enveloppe nucléaire interne. C'est sans doute la cause de l'épaississement apparent de la membrane nucléaire en coupe semi-fine (PI. 3-1 et 3-2). La membrane nucléaire peut montrer quelques bourgeonnements ou invaginations. Les très gros nucléoles sont réticulés et situés au centre du noyau ou non loin de la membrane nucléaire. Ils sont composés de centres clairs fibrillaires et de régions comportant un important matériel très dense. Leur aspect est celui d'un nucléole de cellule à forte activité de synthèse.

Les mitochondries sont sphériques avec des crêtes fines et parallèles, leur matrice présente des régions claires. Peu nombreuses, elles sont à ce stade polarisées et souvent proches de I'appareil de Golgi (PI. 1-1a et 1b). Ce dernier est

\section{PLANCHE 2}

FIG. 1a et 1b. - Zygotène $(19,25$ j p.c.). Composés latéraux des filaments chromosomiques groupés par paire (CL) avec polarisation, fine enveloppe nucléaire (EN), nucléole (Nu), mitochondries $(\mathrm{m})$. $X: 5000$.

FIG. 2a et $2 \mathrm{~b}$. - Pachytène $(21,25 \mathrm{j}$ p.c.). Epais composés latéraux répartis dans tout le noyau (CL), importante polarisation de l'appareil de Golgi (Go) et des mitochondries (m). Rassemblement des pores nucléaires (PN). X: 4000 .

FIG. 3a et 3b. - Diplotène (naissance). Amas chromosomiques diffus avec quelques fins filaments, nucléole volumineux, réticulé et sphérique ( $\mathrm{Nu}$ ), vésicules Golgiennes (VGo), mitochondries (m). Dilatation de l'espace périnucléaire (\$). X: 6800 . 
composé de saccules aplatis et de nombreuses petites vésicules circulaires. Ces vésicules, souvent groupées, sont parfois accompagnées de matériel et/ou de granules denses, produits de synthèse ou de digestion. Des « nuages " cytoplasmiques sont trouvés à proximité des mitochondries.

2. Pre/eptotène ( $\mathrm{PI}$. 1-2a et $2 \mathrm{~b} ; 3-3$ et 4 ). - Le noyau « préleptotène " possède des amas épars de chromatine. L'enveloppe nucléaire, apparemment fine et diffuse sur coupes semi-fines, a perdu une grande partie du matériel trouvé condensé contre la membrane au stade précédent ( $\mathrm{PI}$. 3-3 et 4). Les nucléoles, plus petits, restent réticulaires mais fragmentaires. Ils sont composés de régions claires fibrillaires et de matériel dense. Les mitochondries sphériques deviennent plus denses (PI. 1-2a) et sont toujours peu nombreuses. Elles ont perdu leur polarisation avec l'appareil de Golgi et sont maintenant dispersées dans le hyaloplasme. Les crêtes sont parallèles avec quelques régions claires dans la matrice (PI. 3-4). L'appareil de Golgi est peu développé.

3. Leptotène (PI. 1-3a et $3 b ; 3-5,6,7$ et 8 ). - Le noyau au "leptotène » contient de nombreux filaments courts et individualisés. Ce sont les « composés latéraux 》 des chromosomes en cours de formation. A l'extrémité du composé latéral apparaît un dépôt de matériel dense, de forme conique, dont la base vient s'ancrer sur l'enveloppe nucléaire (PI. 3-6 et 3-8). Ce sont les " sites d'attachement " des chromosomes. L'enveloppe nucléaire est très fine ; les deux feuillets sont dépourvus de grains chromatiniens en dehors des sites d'attachement.

Les nucléoles sont des rudiments branchus ou en virgule, composés uniquement de matériel dense. Les mitochondries éparses sont claires ou à moitié vides mais toujours à crêtes fines (PI. 3-6). L'appareil de Golgi, à saccules aplatis ou avec quelques dilatations, demeure inchangé et peu important (PI. 3-7).

\section{PLANCHE 3}

FIG. 1 et 2. - Ovogonie $(17,25$ j p.c.). Nucléole très volumineux et réticulé ( $\mathrm{Nu}$ ) composé de centres clairs fibrillaires (cf) et de régions denses ( $r d)$, dépôt de matériel dense tout le long de l'enveloppe nucléaire $\rightarrow$ avec bougeonnement ou invagination $\rightarrow-1$, pores nucléaires $\rightarrow$ ), mitochondries $(\mathrm{m})$ rondes à crêtes fines et parallèles avec quelques régions claires dans la matrice $(\square)$. $1: X: 11000$ et $2: X: 22000$.

FIG. 3 et 4 . - Préleptotène $(17,75$ j p. c.). Matériel dense contre l'enveloppe nucléaire $\rightarrow$ seulement présent au niveau des amas chromosomiques $(\xi)$.

3 : Nucléole fragmentaire (Nu), centre clair fibrillaire (cf) et région dense ( $r d$ ). $X: 21300$.

4 : Mitochondries $(\mathrm{m})$ avec région claire $(\square)$, vésicules cytoplasmiques (Ve). X : 28500.

FIG. 5-6-7 et 8 . - Leptotène $(18,25$; p. c. $)$.

5 : Rudiment nucléolaire (Nu), enveloppe fine $(\Rightarrow)$, vésicules cytoplasmiques (Ve). X: 16700 .

7: Appareil de Golgi (Go). Nucléole (Nu). X : 13000.

6 et 8 : Composés latéraux de chromosomes $(C L)$ avec dépôt conique de matériel dense aux sites d'attachements à l'enveloppe nucléaire $\leftrightarrow$.

$6: X: 17500$ et $8: X: 32200$.

G : Gonie : PL : Préleptotène ; L : Leptotène ; RE : Réticulum Endoplasmique ; CS : Cellule Somatique. 
4. Zygotène (PI. 2-1a et 1b; 4-1, 2 et 3). - Le stade « zygotène 》 a un nucléoplasme très peu dense aux électrons; les composés latéraux des chromosomes homologues se rapprochent pour former les " paires de chromosomes » et se polarisent à un endroit du noyau formant le stade "bouquet », non loin de l'appareil de Golgi le plus souvent (PI. 2-1a et 1b). Le complexe synaptinémal commence à se former à partir des sites d'attachement à l'enveloppe nucléaire toujours très fine (PI. 4-2 et 3). Quelques dilatations des deux feuillets sont parfois visibles. De nombreux pores nucléaires très groupés sur la membrane nucléaire sont repérables. Cette concentration ne semble pas correspondre à la zone de rassemblement des chromosomes en bouquet (PI. 4-1). Les rudiments nucléolaires sont peu nombreux et peu visibles. Ce ne sont que de courts et fins fragments allongés ou en forme d'anneau, constitués de matériel très dense.

Dans le cytoplasme, le réticulum endoplasmique est constitué de petites vésicules circulaires ou ovales et entourées d'une membrane lisse. Ces vésicules sont réparties dans tout le cytoplasme mais sont toutefois plus nombreuses au voisinage des mitochondries et de l'appareil de Golgi. Ce phénomène sera particulièrement évident au diplotène (PI. 4-7). Les mitochondries sphériques contiennent du matériel dense ou réparti en couches successives. Elles possèdent des crêtes très fines et transversales. Elles sont encore réparties dans tout le cytoplasme mais toutefois plus nombreuses au niveau du développement Golgien qui se trouve dans la région proche des sites d'attachement des chromosomes.

5. Pachytène (PI. 2-2a et $2 b ; 4-4,5$ et 6 ). - Le noyau " pachytène " contient les couples de chromosomes homologues. Le complexe synaptinémal existe sur toute leur longueur. C'est l'épaississement des composés latéraux qui rend ces amas chromosomiques très visibles sur coupes semi-fines. II y a disparition du " stade bouquet " et distribution uniforme des bivalents dans tout le noyau. Le

\section{PLANCHE 4}

FIG. $1-2$ et 3 . - Zygotène $(19,25$ j p.c. $)$.

1 : Nucléole fin et tragmenté $(\mathrm{Nu})$, nombreux pores nucléaires $(\rightarrow)$ sur enveloppe fine $(\Rightarrow)$. $X: 17400$.

2 et 3 : Composés latéraux en cours de rapprochement (CL), formation du complexe synaptinémal (CSy), site d'attachement du chromosome $(\rightarrow)$ à l'enveloppe nucléaire fine $(\Rightarrow)$.

$X: 16700$.

FIG. $4-5$ et 6 . - Pachytène $(21,25 \mathrm{j}$ p.c.). Composés latéraux épaissis (CL), complexe sinaptinémal continu (CSy), site d'attachement chromosomique avec perte du cône dense aux électrons $\rightarrow$.

$X: 17300$.

5: Fragment nucléolaire (Nu). $X: 22100$.

6: Nombreux pores nucléaires $(\rightarrow)$. $X: 7400$.

FIG. 7 et 8 . - Diplotène (naissance).

7 : Contraction des bivalents ( $\mathrm{Bi})$, très nombreuses vésicules sphériques $(\mathrm{Ve})$ et mitochondries $(\mathrm{m})$ denses ou vacuolées $(\mathrm{V})$, pores nucléaires $(\rightarrow)$. X: 9300 .

8: Appareil de Golgi très développé (Go) polarisé à de nombreuses mitochondries (4), saccules dilatés (Sa), complexe synaptinémal (CSy), cellule somatique (CS) avec petites mitochondries allongées $(\mathrm{m})$, réticulum endosplasmique $(\mathrm{RE}) . X: 7700$.

CG : Cellule Germinale ; $Z$ : Zygotène ; $P$ : Pachytène ; $D$ : Diplotène ; $m$ : mitochondries ; Ve : vésicules. 

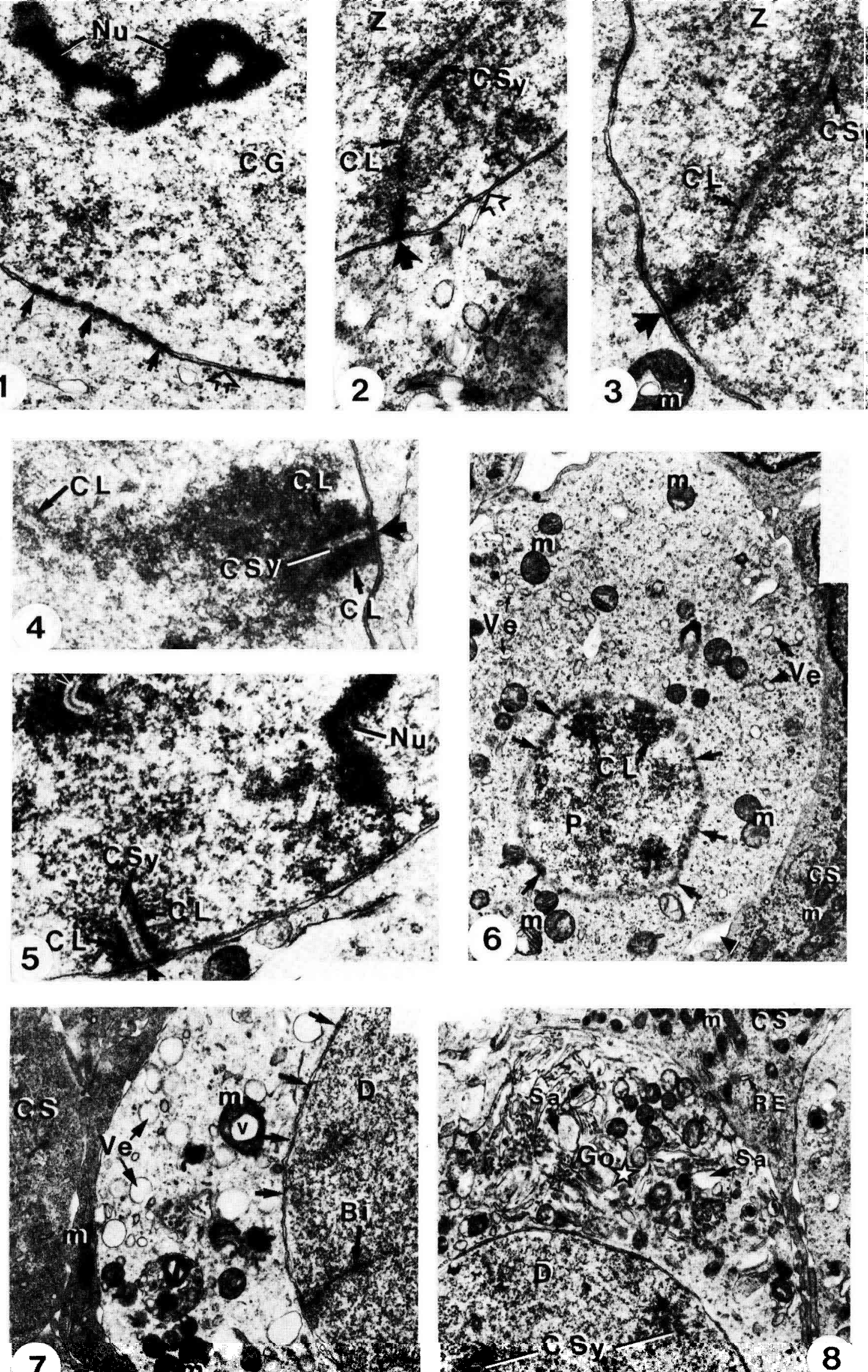

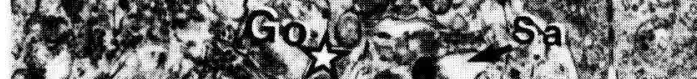

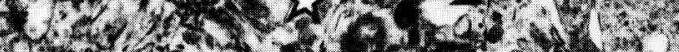

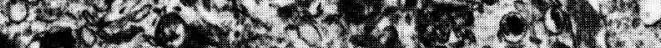

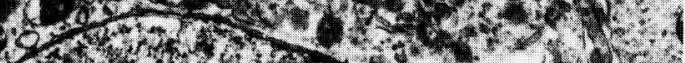

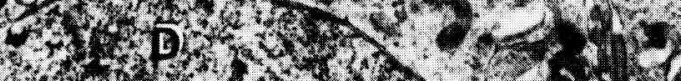

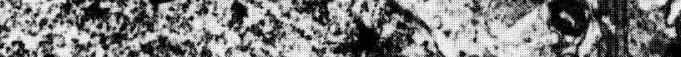

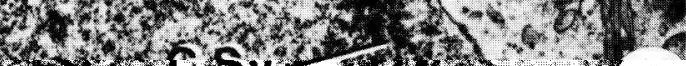

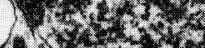

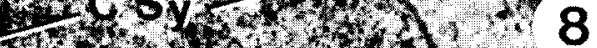


cône dense aux électrons des sites d'attachement à l'enveloppe nucléaire, présent aux stades leptotène et zygotène, va disparaître au cours du pachytène ( $\mathrm{PI}$. 4-4 et 5). Le nucléole est constitué de longs fragments de matériel très dense. II amorce un début de croissance.

Le cytoplasme contient, outre de nombreux polyribosomes, de nombreuses petites vésicules à membrane lisse, rondes ou allongées, de type Golgien (PI. 4-6). L'appareil de Golgi devient particulièrement développé avec des saccules très dilatés. Les mitochondries sont, soit denses avec peu de crêtes, soit claires avec des crêtes parallèles et quelquefois élargies. Elles sont encore éparses dans le cytoplasme au début du stade pachytène, mais se regroupent près de I'appareil de Golgi pour former une importante polarisation dans une région du cytoplasme en fin de pachytène (PI. 2-2a).

6. Diplotène (PI. 2-3a et $3 b ; 4-7$ et 8 ). - Le nucléoplasme au "diplotène » redevient plus clair avec quelques amas très diffus de chromatine. La contraction des bivalents est maximale à ce stade, ce qui les rend peu différents des monovalents du stade leptotène. Quelques complexes synaptinémaux restent encore visibles en début de stade (PI. 4-7 et 8).

L'enveloppe nucléaire, fine, présente fréquemment des dilatations de l'espace périnucléaire (PI. 2-3a et $3 \mathrm{~b}$ ). La taille du nucléole augmente considérablement ; celui-ci redevient réticulé et sphérique et se retrouve en bordure de la membrane nucléaire (PI. 2-3a).

Le cytoplasme est clair avec de très nombreuses petites vésicules sphériques. L'appareil de Golgi est tout à fait remarquable et très développé avec de nombreux saccules dilatés (PI. 4-7 et 8). La polarisation de mitochondries accompagnant l'appareil de Golgi est réapparue ; toutefois, des mitochondries sont aussi réparties autour du noyau. Elles sont denses ou plus claires avec quelques vacuoles et des crêtes légèrement dilatées.

PLANCHE 5. - Répliques cellulaires d'ovaires de rat après cryofracture

FIG. 1. - A 17,25 j p.c. (stade " dominant »: ovogonie) : dispersion régulière de pores nucléaires $(P N)$ isolés ou en petits groupes sur toute la membrane nucléaire. $X: 11900$.

FIG. 2. - A 17,75 j p.c. (stade " dominant " : préleptotène) : dispersion régulière de paquets plus riches en pores nucléaires (PN). $X: 14900$.

FIG. 3. - A 19,25 j p.c. (stade " dominant »: zygotène) : importante polarisation de l'ensemble des pores nucléaires (PN). X: 7800 .

FIG. 4. - A 21,25 j p.c. (stade " dominant » : pachytène) : zone plus étendue de forte concentration de pores nucléaires (PN). (dil) dilatation périnucléaire. $X: 9400$.

FIG. 5. - Mitochondries sphériques $(m)$ de cellules germinale riches en particules intramembranaires. $X: 51000$.

FIG. 6. - Mitochondrie (m) allongée de cellule somatique (CS), (PN) pores nucléaires répartis très régulièrement. $X: 29400$.

FIG. 7. - Pores nucléaires (PN) de cellule germinale avec accumulation de particules intramembranaires dans le pore sur la face exoplasmique de la membrane nucléaire externe (MEe), répartition identique des particules dans le pore et entre les pores sur la face protoplasmique de la membrane nucléaire interne (Mlp). X : 54000.

CYT : Cytoplasme ; $m$ : mitochondrie ; MIp : membrane nucléaire interne face protoplasmique ; MEe : membrane nucléaire externe face exoplasmique $; \rightarrow$ : angle d'ombrage de la fracture. 


\section{Modification de la membrane nucléaire des cellules germinales.}

La cryofracture est la meilleure méthode pour rendre compte des changements de la répartition dans l'espace des pores nucléaires des cellules, répartition difficile à décrire en ultrastructure classique sur coupes uitrafines.

Les cellules germinales ont été identifiées sur les répliques, d'une part, par la forme particulièrement sphérique de leur noyau et par la taille de celui-ci nettement supérieure à celle de l'ensemble des autres noyaux et d'autre part, grâce à une caractéristique cytoplasmique spécifique de ces cellules. Les mitochondries y sont rondes et volumineuses (PI. 5-5) alors que les mitochondries sont allongées dans les cellules somatiques (PI. 5-6).

La localisation des pores nucléaires apparaît avec les caractéristiques suivantes sur les répliques (fig. $1,2,3,4,5$ ) :

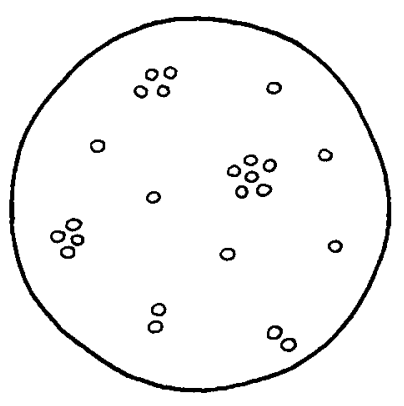

FIG. 1. - A 17,25 j d'âge post-coïtum (stade "dominant » ovogonie) : sur toutes les membranes nucléaires des cellules germinales, il est observé une dispersion très régulière des pores nucléaires, isolés ou le plus souvent en petits groupes de 6 à 8 pores (PI. 5-1).

PLANCHE 6. - Liaisons particulières entre cellules somatiques d'ovaire de rat après cryofracture

FIG. 1. - Cellule somatique (CS) d'ovaire à 19,25 j p.c. : longues jonctions imperméables (ji) très nombreuses sur la membrane plasmique (MP). Jonction perméable visible $(\rightarrow)$; pores nucléaires (PN) nombreux et distribués régulièrement. (MIp) membrane nucléaire interne face protoplasmique, (MEe) membrane nucléaire externe face exoplasmique. $X: 13600$.

FIG. 2. - Membrane plasmique de cellule somatique d'ovaire à 18,25 j p.c. Jonctions imperméables (ji) et jonctions perméables (jp). X : 50000.

FIG. 3. - Membranes plasmiques de deux cellules somatiques accolées (1 et 2) d'ovaire à 21,25 j p.c. La fracture fait apparaître: la face protoplasmique (Face P) de la cellule 1 avec les particules intramembranaires en relief au niveau des jonctions imperméables (ji) et perméables (jp) et la face exoplasmique (Face E) de la cellule 2 avec les particules en creux au niveau des jonctions (ji et jp). X : 50000 .

FIG. 4. - Cellule somatique (CS) d'ovaire à $4 \mathrm{j}$ p.n. Nombreuses jonctions perméables (jp) et imperméables (ji), (NP) pores nucléaires. $X: 13400$.

FIG. 5. - Membrane plasmique (MP) de cellule somatique d'ovaire à 15,25 j p.c. : face exoplasmique (E), lignes de jonctions imperméables (ji) peu nombreuses, groupées à de petites jonctions perméables (jp). X: 47200.

FIG. 6. - Importante jonction perméable (jp) sur la face protoplasmique (P) d'une cellule somatique d'ovaire à $4 \mathrm{j}$ p.n. et sur la face exoplasmique (E) de la cellule somatique voisine. Distribution particulière en mosaïque des particules intramembranaires au niveau des jonctions perméables (jp) et alignement continu de celles-ci dans les jonctions imperméables (ji). X : 64700 .

$\rightarrow$ angle d'ombrage de la fracture. 
FIG. 2. - A 17,75 j d'âge post-coïtum (stade "dominant " préleptotène) : il est constaté que, sur la membrane nucléaire de la majorité des cellules germinales, sont présents des paquets plus riches en pores (10 à 20). La distribution se fait sur toute la surface nucléaire (PI. 5-2).

FIG. 3. - A 18,25 j d'âge post-coïtum (stade " dominant " leptotènel: les régions de membrane nucléaire présentant des pores sont maintenant séparées les unes des autres par des zones qui en sont totalement dépourvues. II y a maintenant de 20 à 40 pores par paquet.

FIG. 4. - A 19,25 j d'âge post-coïtum (stade " dominant " zygotène) : une importante polarisation de l'ensemble des pores nucléaires ( $p /$ us de 100) apparaît dans une région déterminée du noyau; il n'y a plus de pores en dehors de cette zone (PI. 5-3).
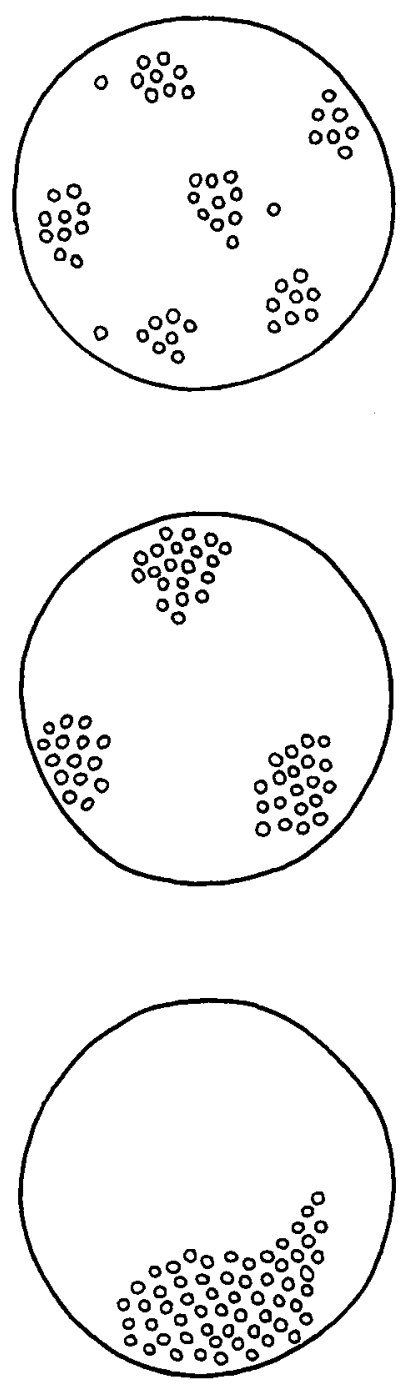

FIG. 5. - A 21,25 j d'âge post-coïtum et à la naissance (stades " dominants 》: pachytène et diplotène) : la zone de forte concentration des pores nucléaires devient plus étendue (PI. 5-4).

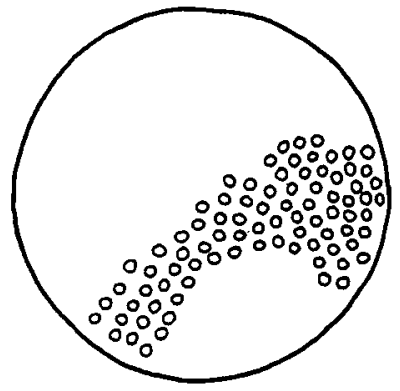


II n'a pas été noté de différences particulières de structure du pore nucléaire au cours de nos observations ( $\mathrm{PI}$. 5-7). Nous pouvons faire la description générale suivante de ces pores :

- il y a une accumulation de particules membranaires sur la face exoplasmique de la membrane nucléaire externe avec peu de particules entre les pores ;

- par contre, sur la face protoplasmique de la membrane nucléaire interne, les particules sont réparties de la même façon dans le pore et entre les pores nucléaires ;

- l'image de la bordure du pore est différente selon qu'il s'agit de la membrane nucléaire exoplasmique ou de la membrane nucléaire protoplasmique, externe ou interne de l'enveloppe (PI. 5-7).

\section{Liaisons entre cellules somatiques.}

La cryofracture permet d'étudier la structure fine des éléments participant aux jonctions entre cellules au niveau de leurs membranes plasmiques. La disposition des particules intramembranaires qui les constituent présente une organisation très particulière. II existe :

- soit, des particules alignées pour former des "jonctions imperméables ",

- soit, des particules rassemblées en de larges zones et formant une véritable mosaïque par la régularité de leur distribution ; ce sont alors des « jonctions perméables $\$$.

Nous avons noté la présence de longues jonctions imperméables sur les membranes plasmiques des cellules somatiques bordant les cellules germinales avec :

- une à deux lignes jonctionnelles dans les jeunes ovaires embryonnaires de 15,25 j p.c. (PI. 6-5),

- plusieurs lignes orientées en tous sens dans les ovaires à partir de 18,25 j p.c. (PI. 6-1, 2, 3 et 4).

Par ailleurs, sur ces mêmes membranes plasmiques de cellules somatiques, les plaques de particules intramembranaires de jonctions perméables sont :

- disposées régulièrement, isolées et petites dans les jeunes ovaires embryonnaires de 15,25 j p.c. (PI. 6-5),

- associées aux longues jonctions imperméables dans les ovaires à partir de $18,25 \mathrm{j}$ p.c. Ce phénomène s'accentue à $21,25 \mathrm{j}$ p.c. Ce sont les jonctions perméables qui s'incorporent à la barrière imperméable (PI. 6-3 et 6).

\section{Discussion.}

Les études en microscopie électronique des différents types de cellules germinales femelles, durant les premiers stades de la prophase méiotique, sont nombreuses chez le rat (Sotelo, 1959 ; Odor, 1960 ; Franchi et Mandl, 1962), la souris (Odor et Blandau, 1969 ; Wassarman et Josefowicz, 1978), le hamster (Weakley, 
1967), le lapin (Gondos et Zamboni, 1969), I'homme (Stegner et Wartenberg, 1963 ; Baker et Franchi, 1967), d'autres mammifères (Zamboni, 1972; Gondos, 1978) et les insectes (Palevody, 1973; Rasmussen, 1976).

La description des éléments chromosomiques durant la méiose a porté sur leur changement de structure et de distribution au sein du noyau de la cellule germinale ; ces caractéristiques permettent d'identifier les stades nucléaires et sont les critères fondamentaux de définition des stades d'évolution des cellules germinales au cours de la prophase méïotique (Franchi et Mandl, 1962 ; Vasquez-Nin et Echeverria, 1976; Tsuda, 1965; Mirre et Stahl, 1978 ; Weakley, 1967; Baker et Franchi, 1967, 1972).

Le complexe synaptinémal a également fait l'objet de nombreuses descriptions ultrastructurales au cours des stades leptotène, zygotène et pachytène (Moens, 1969, 1973 ; Stockert et al., 1970 ; Comings et Okada, 1971 ; Esponda et Gimenez-Martin, 1972 ; Schleiermacher et Schmidt, 1973 ; Stack, 1973 ; Chevaillier, 1974 ; Rasmussen, 1976 ; Von Wettstein, 1977). Les observations rapportées ici portent plus particulièrement sur les modifications membranaires et cytoplasmiques.

Des changements dans la distribution de la chromatine sont repérables par des différences de densité aux électrons au sein des noyaux de cellules germinales en méïose. D'une répartition homogène au stade d'ovogonie, la chromatine se condense en de nombreux amas dispersés dans le noyau, certains prenant contact avec l'enveloppe nucléaire pendant le stade préleptotène. Cette distribution a été utilisée pour identifier ce stade (Baker et Franchi, 1972; Bruslé, 1972 ; Mirre et Stahi, 1978).

II est impossible d'assurer que ces localisations de chromatine en contact avec la membrane interne correspondent aux futurs sites d'attachement des chromosomes aux stades leptotène et zygotène.

L'épaississement ou l'affinement de l'enveloppe nucléaire signalé du stade ovogonie au stade dictyotène dépend principalement de la distribution de matériel chromatinien le long de la membrane nucléaire interne : distribution très régulière d'une couche dense tout au long de la membrane interne dans les ovogonies, disparition partielle au cours du préleptotène, complète disparition aux stades leptotène, zygotène et début pachytène puis réapparition en fin de pachytène et surtout au diplotène.

Cet épaississement semble correspondre à la « lamina » dense citée par Stick et Schwarz (1982 et 1983) dans les cellules germinales mâles et femelles. Cette lamina disparaît au cours de la spermatogenèse et du leptotène au pachytène dans les ovocytes. Elle est de nouveau visible au stade diplotène. Au cours des modifications de structure de la lamina, les deux feuillets nucléaires demeurent intacts. Toutefois, quelques dilatations provoquent un élargissement de l'espace périnucléaire. Ces zones de dilatation ont été observées dans des ovocytes de femme et de singe dès le stade préleptotène pour devenir très nombreuses au stade zygotène, pachytène et diplotène (Baker et Franchi, 1969). Ce phénomène de dilatation observé en début de méiose semble être une caractéristique de ces deux espèces. En effet, il n'a pas été vu si précocément chez le rat (Sotelo, 1959 ; Franchi et Mandl, 1962), ni chez la souris (Tsuda, 1965) ou le hamster (Weakley, 
1967), mais seulement en fin de pachytène et surtout au diplotène. II est en relation avec la phase de croissance de l'ovocyte, c'est-à-dire l'augmentation du volume cytoplasmique de la cellule germinale. Cette phase de croissance est déjà amorcée en début de méiose chez l'homme et le singe, mais est plus tardive (fin pachytène) chez le rat. Ces faits expliquent le rôle important que joue l'enveloppe nucléaire dans les échanges de matériaux entre noyau et cytoplasme qui sont nécessaires au cours de la croissance ovocytaire. L'accroissement de ces échanges au sein d'une même cellule est illustré également par la variabilité, en nombre et localisation, des pores nucléaires observés dans les cellules germinales sur coupes ultrafines mais surtout visibles après cryofracture sur les répliques membranaires. Tous les noyaux de cellules somatiques ovariennes présentent des pores nucléaires, peu nombreux et uniformément distribués sur toute la surface de la membrane nucléaire. II n'en est pas de même pour les noyaux des cellules germinales qui montrent des variations d'aggrégation des pores, répartis en régions plus ou moins riches (Bézard et Mauléon, 1981). Avant le début de la prophase méiotique, de petits groupes de pores sont dispersés sur toute la surface nucléaire. Dès l'initiation méiotique, ces groupes deviennent de plus en plus riches et les espaces vides sont de plus en plus grands. Une polarisation de ces pores se produit au cours des stades zygotène et pachytène.

Ces changements, dans leur nombre et leur distribution ont été également décrits chez le mâle au cours de la spermatogenèse (Fawcett et Chemes, 1979). Leur déplacement, au cours de la méiose, correspond à la période de disparition de la lamina dense de l'enveloppe nucléaire, ce qui pourrait expliquer la plus grande mobilité des pores comme suggéré par Fawcett et Chemes (1979) et Troyer et Schwager (1982). Au contraire, le maintien de cette lamelle dans les cellules somatiques et les jeunes ovogonies limiterait leur déplacement; les pores resteraient dispersés régulièrement sur l'enveloppe nucléaire.

La forte concentration de pores nucléaires à des endroits particuliers traduitelle une augmentation réelle de leur nombre ou reflète-t-elle seulement leur mobilité ? Des études sur cellules somatiques ont montré que l'augmentation du nombre de pores dans l'inter-phase est associée à la croissance du volume nucléaire et à la réplication d'ADN avant la division de la cellule (Maul et al., 1972). Des cellules stimulées, à forte activité métabolique et synthétique, augmentent leur nombre de pores nucléaires (Moses, 1964). Une stimulation particulière suivant l'évolution nucléaire de la cellule germinale est peut-être la raison des changements de distribution de ces pores.

Leur formation, leur disparition ou leur déplacement au niveau de l'enveloppe nucléaire d'une cellule, reflètent également les processus métaboliques ou les synthèses de cette cellule (Kessel, 1969 ; Maul et al., 1971). Ces faits peuvent être le reflet d'une activité physiologique différente au cours du déroulement de la prophase méiotique. Par ailleurs, une relation entre la position des pores nuciéaires et l'attachement du chromosome à l'enveloppe nucléaire reste douteuse. Nos résultats en microscopie électronique, de même que ceux de Moses (1960, 1964), montrent qu'ils sont placés entre les chromosomes et qu'ils sont trouvés éloignés des sites de terminaisons du complexe synaptinémal. 
Nos descriptions de la morphologie du nucléole des cellules germinales de rat confirment les observations faites par Franchi et Mandl (1962) et Schuchner (1975). L'ovogonie montre un large nucléole de type réticulé, pourvu de plusieurs centres fibrillaires. Un changement de structure s'amorce dès le préleptotène, préfigurant une diminution de sa taille : il devient fragmentaire et se réduit à quelques corps nucléolaires au leptotène et au zygotène. C'est en fin de pachytène que sa réorganisation et sa croissance reprennent. L'organisation du nucléole et l'étude des synthèses d'ARN et d'ADN ont fait l'objet de nombreuses descriptions ultrastructurales (rat: Schuchner, 1975 ; chat : Morato, 1965 ; champignons : Stockert et al., 1970 ; plantes : La Cour, 1975 ; souris et caille: Mirre et Stahl, 1976, 1978 ; homme : Très, 1975 ; Stahl et al., 1978, 1980 ; Hartung et al., 1979 ; Mirre et al., 1980). Cette évolution des composés nucléolaires est en relation avec les synthèses nucléaires. En effet, la fragmentation du nucléole correspondrait à l'arrêt des synthèses d'ARN qui reprendraient dans le noyau, en fin pachytène, au moment où a lieu le regroupement des constituants nucléolaires (Mirre et Stahl, 1978).

Des modifications de structure et de localisation d'organelles cytoplasmiques telles que les mitochondries reflètent également des changements dans le métabolisme de la cellule germinale. Nous n'avons prêté aucune attention particulière aux mitochondries, mais nous avons noté des phénomènes de polarisation dans les ovogonies âgées peu avant le début de la prophase méiotique, puis de dispersion dès l'entrée en méiose et enfin de nouvelle polarisation en fin de pachytène. Ces rassemblements de mitochondries correspondent peut-être aux regroupements décrits dans le spermatocyte de rat à cette même période d'évolution de la cellule germinale et sont peut-être en relation avec la synthèse d'ARN mitochondrial au cours des divisions de la méiose (Curgy, 1972 ; André, 1962 ; De Martino et al., 1979). Les quelques études des mitochondries réalisées chez la femelle (lapin : Anderson, 1968 ; Anderson et al., 1970) n'ont pas été faites en relation avec un stade nucléaire défini de la prophase méiotique.

L'évolution tout à fait remarquable de la structure de l'appareil de Golgi en fin de pachytène avec augmentation du nombre de vésicules et dilatation des saccules, indique également des modifications métaboliques susceptibles de se produire au cours de la gamétogenèse (Young, 1973). Elles ont été étudiées dans les cellules de Sertoli chez le rat (Rambourg et al., 1979) et dans les cellules germinales d'ovaire de chauve-souris (Ruby et Webster, 1972). L'appareil de Golgi participe au processus de "secrétion " de polysaccharides et de polypeptides qui contribuent au renouvellement de la membrane cellulaire. L'augmentation de taille de l'ovocyte se produisant au début du stade diplotène nécessite un tel renouvellement de la membrane plasmique.

Les échanges de matériel et d’informations nécessaires à la différenciation cellulaire ne se font pas uniquement entre noyau et cytoplasme d'une même cellule mais également entre cellules voisines. Ils se produisent par l'intermédiaire de jonctions mises en évidence sur les membranes plasmiques après cryofracture des ovaires embryonnaires.

Pour les liaisons repérées sur les cellules somatiques qui bordent les cellules germinales, une évolution de structure peut être proposée : 
- une mauvaise étanchéité des membranes est constatée dans les ovaires avant l'apparition de la méiose, caractérisée par la présence de seulement une ou deux lignes de jonctions imperméables.

- la juxtaposition de plusieurs lignes jonctionnelles au moment de l'apparition de la méiose interdit ou restreint les échanges entre cellules. De ce fait, un groupe de cellules peut se maintenir dans un environnement interne, différent du groupe voisin.

Les jonctions perméables de petite taille sont rencontrées dispersées sur les membranes plasmiques provenant de jeunes gonades ; elles ont de plus grandes surfaces et sont liées aux jonctions imperméables à partir de $18,25 \mathrm{j}$ p.c. Ces structures de jonctions perméables, nommées " gap junctions ", participent au transfert direct des messages chimiques entre cellules (Berkaloff et al., 1977). L'évolution de leur distribution est en accord avec la formation au sein de l'ovaire des cordons ovigères qui vont tendre à s'individualiser.

Ces observations sont à rapprocher de celles réalisées au niveau des cellules de Sertoli de jeunes testicules (homme : Camatini et al., 1979 ; chien : Connell, 1975, 1976, 1978 ; rat : Nagano, 1980) où des jonctions imperméables sont signalées, ainsi que des jonctions perméables dans des testicules embryonnaires de souris (Nagano et Suzuki, 1978).

Par contre, plus tard dans l'organogenèse, d'importantes jonctions perméables ont été localisées sur les membranes plasmiques des cellules de la granulosa de follicule préovulatoire chez le rat (Amsterdam et al., 1976; Burghardt et Anderson, 1981). Elles sont la preuve d'échanges de matériel et d'informations entre ces cellules qui contribuent à la croissance folliculaire et à la maturation ovocytaire.

Cette étude morphologique suggère d'importants changements des synthèses d'acides nucléiques et de protéines et des échanges de matériel et d'informations entre noyau et cytoplasme, ou entre cellules. Une étude biochimique des ovocytes en prophase méiotique serait nécessaire. Cette étude implique la mise au point de techniques de séparation des cellules germinales et des cellules somatiques, techniques encore difficilement applicables au tissu ovarien.

Reçu en mars 1984. Accepté en juin 1984.

\section{Références}

AMSTERDAM A., JOSEPHS R., LIEBERMAN M. E., LINDNER H. R., 1976. Organization of intramembrane particules in freeze-cleaved gap junctions of rat Graafian follicles : optical diffraction analysis. J. Cell Sci, 21, 93-105.

ANDERSON E., 1968. The structure of mitochondries during oogenesis and early embryogenesis in the rabbit. Anat. Rec., 160. 307.

ANDERSON E., CONDON W., SHARP D., 1970. A study of oogenesis and early embryogenesis in the rabbit, Oryctolagus cuniculus, with special reference to the structural changes of mitochondria. J. Morph., 130, 67-92. 
ANDRE J., 1962. Contribution à la connaissance du chondriome. Etude de ses modifications ultrastructurales pendant la spermatogenèse. J. Uitrastr. Res., Suppl. 3, 185.

BAKER T. G., FRANCHI L. L., 1967. The fine structure of oogonia and oocytes in human ovaries. J. Cell Sci., 2, 213-224.

BAKER T. G., FRANCHI L. L., 1969. The origin of cytoplasmic inclusions from the nuclear envelope of mammalian oocytes. Z. Zellforsch. mikrosk. Anat., 93, 45-55.

BAKER T. G., FRANCHI L. L., 1972. The fine structure of oogonia in the Rhesus Monkey (Macaca mulattal. Z. Zellforsch. mikrosk. Anat, 126, 53-74.

BERKALOFF A., BOURGUET J., FAVARD P., LACROIX J. C., 1977. Biologie et physiologie cellulaire l. Editeur Hermann, Paris.

BÉZARD J., MAULÉON P., 1981. Ultrastructural changes in mammalian oocytes at the beginning of meiotic prophase with special reference to the nuclear pores studied by freeze fracture technique, 93-103. In A. G. BYSKOV, H. PETERS, Development and function of the reproductive organs. Excerpta med., Amsterdam, Oxford, Princeton.

BÉZARD J., MAULÉON P., 1984. Evolution des cellules germinales femelles au cours de la prophase méiotique chez le rat : critères de reconnaissances cytoplasmique et nucléaire des différents stades en histologie fine. Reprod. Nutr. Dévelop., 24, 633-654.

BRUSLÉ J., 1972. Les infrastructures germinales femelles précoces (gonocytes, ovogonies et ovocytes 1). Ann. Biol., 11, 505-571.

BURGHARDT R. C., ANDERSON E., 1981. Hormonal modulation of gap junctions in rat ovarian follicles. Cell Tiss. Res., 214, 181-193.

CAMATINI M., FRANCHI E., de CURTIS I., 1979. Sertoli junctions in human testes : a freeze fracture and lanthanum tracer study. J. submicr. Cytol., 11, 511-516.

CHEVAILLIER P., 1974. Recherches sur le complexe synaptonématique. I. Mise en évidence des constituants nucléoprotéïques du complexe synaptonématique par la méthode de Bernhard. J. Microsc., 19, 147-154.

COMINGS D. E., OKADA T. A., 1971. Whole mount electron microscopy of human meiotic chromosomes. Exp. Cell Res., 65, 99-103.

CONNELL C. J., 1975. A freeze fracture and lanthanum tracer study of the junction between the Sertoli celis of the dog. Anat. Rec., 181, 336-337.

CONNELL C. J., 1976. A freeze fracture and lanthanum tracer study of the development of the junction between Sertoli cells of the prepubertal dog. J. Cell Biol., 70, 238.

CONNELL C. J., 1978. A freeze fracture and lanthanum tracer study of the complex junction between Sertoli cells of the canine testis. J. Cell Biol., 76, 57-75.

CURGY J. J., 1972. Mitochondrial RNA synthesis during meiotic divisions in Locust male germ cells. Exp. Cell Res., 75, 127-137.

DE MARTINO C., FLORIDI A., MARCANTE M. L., MALORN! W., SCORZA-BARCELLONA P., BELLOCI M., SILVESTRINI B., 1979. Morphological, histochemical and biochemical studies on germ cell mitochondria of normal rats. Cell Tiss. Res., 196, 1-22.

ESCAIG J., NICOLAS G., 1976. Cryofractures de matériel biologique réalisées à très basses températures en ultra-vide. C. R. Acad. Sci. Paris, Sér. D, 283, 1245-1248.

ESPONDA P., GIMENEZ-MARTIN G., 1972. The attachment of the synaptonemal complex to the nuclear envelope. An uitrastructural and cytochemical analysis. Chromosoma, 38, 405-417.

FAWCETT D. W., CHEMES H. E., 1979. Changes in distribution of nuclear pores during differentiation of the male germ cells. Tiss. Cell., 11, 147-162.

FRANCHI L. L., MANDL A. M., 1962. The ultrastructure of oogonia and oocytes in the foetal and neonatal rat. Proc. roy. Soc. Lond. B, 157, 99-114.

GONDOS B., 1978. Oogonia and oocytes in mamma/s, p. 83. In R. E. JONES, The vertebrate ovary. Plenum Press, New York and London.

GONDOS B., ZAMBONI L., 1969. Ovarian development. The functional importance of germ cell interconnections. Fertil. Steril., 20, 176-189.

HARTUNG M., MIRRE C., STAHL A., 1979. Nucleolar organizers in human oocytes at meiotic prophase 1 studied by the silver N.O.R. method and electron microscopy. Hum. Genet., 52 , 295-308.

KESSEL P. G., 1969. Fine structure of the pore annulus complex in the nuclear envelope and annulate lamellae of germ cells. Z. Zellforsch. mikrosk. Anat., 94, 441-453. 
LA COUR L. F., 1975. The nucleolus at prophase of meiosis in three plants ; an ultrastructural study. Proc. roy. Soc. Lond. B., 191, 231-243.

MAUL G. G., MAUL H. M., SCOGNA J. E., LIEBERMAN M. W., STEIN G. S., HSU B. Y., BORUN T. W., 1972. Time sequence of nuclear pore formation in PHA stimulated lymphocytes and in Hela cells during the cell cycle. J. Cell Biol., 55, 433.

MAUL G. G., PRICE J. W., LIEBERMAN M. W., 1971. Formation and distribution of nuclear pore complexes in interphase. J. Cell Biol., 51, 405-418.

MIRRE C., HARTUNG M., STAHL A., 1980. Association of ribosomal genes in the fibrillar center of the nucleolus : a factor influencing translocation and non disjunction in the human meiotic oocyte. Proc. nat. Acad. Sci., USA, 77, 6017-6021.

MIRRE C., STAHL A., 1976. Ultrastructural study of nucleolar organizers in the quail oocyte during meiotic prophase I. J. U/trastr. Res., 56, 186-201.

MIRRE C., STAHL A., 1978. Ultrastructure and activity of the nucleolar organizer in the mouse oocyte during meiotic prophase. J. Cell Sci., 31, 79-100.

MOENS P. B., 1969. The fine structure of meiotic chromosome polarisation and pairing in Locusta migratoria spermatocytes. Chromosoma, 28, 1-25.

MOENS P. B., 1973. Mechanisms of chromosome synapsis at meiotic prophase. Int. Rev. Cyto/., 35. $117-134$.

MORATO M. J., 1965. L'ultrastructure du nucléole des ovocytes de chat et son involution au cours de la prophase méiotique. C. R. Soc. Biol., 159, 1633.

MOSES M. J., 1960. Breakdown and reformation of the nuclear envelope at cell division. 4th intern. Conf. Electr. Microsc. Proc., Berlin (1958), 230-233, Springer-Verlag.

MOSES M. J., 1964. The nucleus and chromosomes, 423-558. In G. H. BOURNE Cytology and cell physiology,3rd Edn, Acad. Press, New York.

NAGANO T., 1980. Freeze fracture observations on the rat Sertoli cell junctions by metal contact freezing. J. Electron. Microsc., 29, 250-255.

NAGANO T., SUZUKI F., 1978. Cell to cell relationships in the seminiferous epithelium in the mouse embryo. Cell Tiss. Res., 189, 389-401.

ODOR D. L., $1960 . \quad$ Electron microscopic studies on ovarian oocytes and unfertilized tubal ova in the rat. J. biophys. biochem. Cytol., 7, 567-574.

ODOR D. L., BLANDAU R. J., 1969. Ultrastructural studies on fetal and early postnatal mouse ovaries. I. Histogenesis and organogenesis. Am. J. Anat., 124, 163-186.

PALEVODY C., 1973. Différenciation du noyau de l'ovocyte au cours de la prophase méiotique chez les Collemboles (Insectes, Aptérygotes). Etude ultrastructurale. C. R. Acad. Sci. Paris, Sér. D, 277, 2201-2204.

RAMBOURG A., CLERMONT Y., HERMO L., 1979. Three dimensional architecture of the Golgi apparatus in Sertoli cells of the rat. Am. J. Anat., 154, 455-476.

RASMUSSEN S. W., 1976. The meiotic prophase in Bombyx mori femaies analyzed by three dimensional reconstructions of synaptonemal complexes. Chromosoma, 54, 245-293.

RUBY J. R., WEBSTER R. M., 1972. Origin of the Golgi complex in germ cells in the developing ovary of the rat. Z. Zellforsch. mikrosk. Anat., 133, 1-12.

SCHLEIERMACHER E., SCHMIDT W., 1973. Changes of synaptonemal complex at the end of pachytene. Hum. Genet., 19, 235-245.

SCHUCHNER E. B., 1975. Ultrastructural evolution of nucleoli of female rat germ cells. Fertil. Steril., 26, 924-931.

SOTELO J. R., 1959. An electron microscopic study on the cytoplasmic and nuclear components of rat primary oocytes. Z. Zellforsch. mikrosk. Anat., 50, 749-765.

STACK S., 1973. The synaptonemal complex and the achiasmatic condition. J. Cell Sci., 13, 83-95.

STAHL A., MIRRE C., HARTUNG M., KNIBIEHLER B., NAVARRO A., 1978. Localisation and structure of nucleolar organizers in the oocyte during meiotic prophase I. Ann. Biol. anim. Bioch. Biophys., 18, 399-408.

STAHL A., MIRRE C., HARTUNG M., KNIBIEHLER B., $1980 . \quad$ Localisation, structure et activité des gènes ribosomiques dans le nucléole de l'ovocyte en prophase de méiose. Reprod. Nutr. Dévelop., 20, 469-483.

STEGNER H. E., WARTENBERG G., 1963. Electron microscopic studies on human oocyte at different stages of oogenesis. Arch. Gynäk., 199, 151-172.

Reproduction, Nutrition, Développement, $n^{\circ} 6,1984 .-6$. 
STICK R., SCHWARZ H., 1982. The disappearance of the nuclear lamina during spermatogenesis : an electron microscopic and immunofluorescence study. Cell Diff., 11, 235-243.

STICK R., SCHWARZ H., 1983. Disappearance and reformation of the nuclear lamina structure during specific stages of meiosis in oocytes. Cell, 33, 949-958.

STOCKERT J. C., GIMENEZ-MARTIN G., SOGO J. M., 1970. Nucleolus and synaptonemal complexes in pachytene meiocytes of Allium Cepa. Cytobiologie, 2, 235-250.

TRES L. L., 1975. Nucleolar RNA synthesis of meiotic prophase spermatocytes in the human testis. Chromosoma, 53, 141-151.

TROYER D., SCHWAGER P., 1982. Evidence for nuclear membrane fluidity : proacrosome migration and nuclear pore redistribution during grasshopper spermiogenesis. Cell Motil., 4, 355-367.

TSUDA H., 1965. An electron microscope study on the oogenesis in the mouse with special reference to the behaviors of oogenia and oocytes at meiotic prophase. Arch. histol. jap., 25, 533-555.

VASQUEZ-NIN G. H., ECHEVERRIA O. M., 1976. Ultrastructural study on the meiotic prophase nucleus of rat oocytes. Acta anat., 96, 218-231.

WETTSTEIN D. (von), 1977. The assembly of the synaptinemal complex. Phil. Trans. roy. Soc., Lond. B, 277, 235-243.

WASSARMAN P. M., JOSEFOWICZ W. J., 1978. Oocyte development in the mouse : an ultrastructural comparison of oocytes isolated at various stages of growth and meiotic competence. J. Morph., 156, 209-236.

WEAKLEY B., 1967. Light and electron microscopy of developing germ cells and follicle cells in the ovary of the golden hamster : twenty-four hours before birth to eight days post-partum. $J$. Anat., 101, 435-459.

YOUNG R. W., 1973. The role of the Golgi complex in sulfate metabolism. J. Cell Biol., 57, 175-189.

ZAMBONI L., 1972. Comparative studies on the uitrastructure of mammalian oocytes, 5-45. In J. BIGGerS, A. W. SHUETZ, Oogenesis, Univ. Park. Press, Butterworths, London. 